\title{
Hard numbers? The long-term decline in violence reassessed. Empirical objections and fresh perspectives - Corrigendum
}

Gerd Schwerhoff, Benjamin Seebröker, Alexander Kästner and Wiebke Voigt

In the original publication of this article an incorrect source was given for Figure 3. Footnote 129 which cites the source of the figure should read: From: Eisner, 'Long-term historical trends', 98.

Schwerhoff, G., Seebröker, B., Kästner, A., \& Voigt, W. (2021). Hard numbers? The long-term decline in violence reassessed. Empirical objections and fresh perspectives. Continuity and Change 36 (1), 1-32. doi:10.1017/S0268416021000096 